\title{
Determination of the Population Allelic Frequency of the Variants of the MPS Complex in Southwestern Colombia
}

Journal of Inborn Errors of Metabolism \& Screening 2021, Volume 9: e20210021 DOI: https://doi.org/10.1590/2326-4594JIEMS-2021-0021

\author{
Lina Johanna Moreno Giraldo',2,3,4 (i), José María Satizabal ${ }^{1,2,4}$ (D) \\ and Adalberto Sánchez Gómez ${ }^{1,2,4}$
}

\begin{abstract}
Mucopolysaccharidoses are lysosomal storage diseases characterized by the excessive accumulation of glycosaminoglycan sulfate in organs and tissues. To determine the population allelic frequency of the MPS complex variants in a population without clinical and molecular diagnosis of MPS. An observational descriptive study was carried out where the allelic frequency of variants presents in the IDUA, IDS, SGSH, NAGLU, HGSNAT, GNS, GALNS, GLB1, ARSB, GUSB, HYAL1 genes was determined by means of the sequencing of 320 exomes from patients without a clinical diagnosis of MPS; the results were tabulated, and allelic frequency formulas were used to determine the values associated with each of the genes. 509 alleles associated with the MPS complex were reported, of which 262 have not been previously reported. Genes with the most frequent allelic presence were IDUA, GLB1 and GALNS, involved in MPS I and MPS IV A / B. The total frequencies ranged between 0.00393 (2 alleles) and 0.47937 (248 alleles). These studies make it possible to determine polymorphisms that circulate in the country, present in patients not affected with MPS, allowing to expand the knowledge about the characteristics of the alleles that are present in affected patients.
\end{abstract}

Keywords: Computational Biology, Exome sequencing, Mucopolysaccharidosis Complex, Lysosomal Storage Diseases, Allelic Frequency.

\section{Introduction}

Mucopolysaccharidosis complex (MPS) is a group of orphan diseases - rare, of low prevalence, characterized by the deficiency of enzymes involved in the metabolism of glycosaminoglycans (GAGs) at lysosomal level [1]. It is characterized by intracellular GAG accumulation, producing alterations of multiple organs and systems. The clinical presentation in patients with MPS includes multisystemic affectation, mainly the liver, the spleen, the central nervous system, bone, cartilages, eyes and, when stored in excessive quantities, developmental problems, skeletal abnormalities, cervical myelopathy, and spinal cord compression, defects in the immune and neural systems and death at an early age are presented $[2,3]$.

Seven different types of MPS disorders are recognized (I, II, III, IV, VI, VII, and IX) with 11 specific lysosomal enzyme deficiencies. All types of MPS have an autosomal recessive mode of inheritance, except for MPS II (Hunter syndrome), which is X-linked recessive inherited, and therefore, affects males primarily [4]. Overall, the prevalence is 1.04 to $4.8 / 100,000$ births, varying by country, region, or ethnic background [5].
In Colombia, the study conducted by Gómez et al. (2012) estimated that the combined frequency of all MPS cases is 1.98 cases per 100,000 live births, with type IV being the highest frequency with 0.68 per 100.000 live births; they also proposed an incidence of $0.45: 100,000(\mathrm{VN})$ in patients with MPS I; 0.17:100,000 (VN) in MPS III; 0.68:100,000 (VN) in MPS IVA; $0.23: 100,000(\mathrm{VN})$ in patients with MPS VI; there were no incidences reported for MPS VII and MPS IX. Considering data until 2012, the authors also determined that in Colombia,

\footnotetext{
${ }^{1}$ Universidad del Valle, Facultad de Salud, Cali, Valle del Cauca, Colombia.

2 Universidad Santiago de Cali, Valle del Cauca, Colombia.

${ }^{3}$ Universidad Libre, Cali, Valle del Cauca, Colombia.

${ }^{4}$ Universidad del Valle, Grupo de Enfermedades Congénitas del Metabolismo, Cali, Colombia.
}

Received April 25, 2021, and in revised form May 20, 2021. Accepted for publication July 05, 2021.

\section{Corresponding Author:}

Lina Johanna Moreno Giraldo, E-mail: linajohannamoreno@yahoo.es 
MPS IVA or Morquio A syndrome has a frequency of 0.68 cases per 100.000 live births, with MPS being more frequent in the Colombian population [6].

According to the report of the weekly epidemiological bulletin 05 for the epidemiological week from January $27^{\text {th }}$ to February $2^{\text {nd }}$ in 2019, Colombia, the prevalence of MPS IV was presented with a sample size of approximately 150 patients, corresponding to $0.3: 100,000$ inhabitants. For the epidemiological period IV of 2020 , the ratio of notification for MPS II was 7 cases $(0.15 \%)$, and MPS VI was 6 cases $(0.13 \%)[7,8]$.

In Colombia, it is difficult to measure the frequency of these diseases because only the most severe cases are generally diagnosed, being few mild cases or mutation carriers diagnosed $[9,10]$. For this reason, studies providing complete and updated information associated with the population's allele frequencies of mutations present in the southwestern Colombian population are required. Those will allow the medical community and health authorities the early identification and establishment of diagnosis programs considering that nowadays specific treatment is found for several of them, joint to the importance of the transdisciplinary handling that minimizes the morbidity - mortality attributed to this pathological complex, including appropriate genetic advice and search of possible directed therapeutic targets, allowing an approach to precision medicine.

\section{Methods}

\section{Type of Study}

A descriptive observational study in which the allele frequency of variants identified in the genes IDUA, IDS, SGSH, NAGLU, HGSNAT, GNS, GALNS, GLB1, ARSB, GUSB, and HYAL1, was determined in patients without a clinical diagnosis of MPS. This study is guided by the MPS studies approved in the institutional ethics committee No 016-016, Universidad del Valle.

\section{Study Population}

For the present study, the results gathered from the complete exome sequencing of 320 patients without a clinical diagnosis of MPS from the Southwestern Colombian region, belonging to the database of the 'Instituto de Genética Médica' - GENOMICS (Cali), were taken after signing the consent and informed assent.

\section{Exome Sequencing}

Blood extraction was performed and subsequent collection on filter paper and immersion in phosphate buffer. DNA extraction using Qiagen's DNeasy package. Each sample was quantified, and the DNA quality and quantity were verified. Afterward, massive sequencing of Nextera TM libraries was performed using the Illumina platform with 100X coverage. Alignment with GRCh38 reference genome. The new generation sequencing platform used was Illumina. Modified nucleotides with specific fluorescent labels were used, which presented a chemical modification (reversible terminators) that avoids the union of more than one labeled nucleotide in each reaction site in such a way that it can locate the one that corresponds to each point in the sequence and reducing the risk of errors in the sequencing. The instrument used was HiSeq 2500 machine, with a reading length ( $2 \times 150 \mathrm{bp})$, total bases per sequencing: 450-500 Gb, number of readings (cycles) performed (million) 4000, and coverage $100 \mathrm{X}$. The sequencing results were obtained in VCF files and passed through the first filter of interpretation and prioritization. The annotations include medicament associations TARGET, 1000 genomes, Exome Variant Server, and Exome Aggregation Consortium (ExAC), minor alleles frequencies per population. Variant zygosity, accumulation coverage, and variant allele frequencies are provided when users send VCF files. Variant and gene-level annotations and bioinformatics scores were provided to allow interpretation and prioritization of variants identified in sequencing studies, which included reading frame displacement, insertions/deletions, splice site, missense, and nonsense.

\section{Calculation of Allele Frequencies}

Through the findings of VCFs, variants in the genes associated with the MPS complex were searched. For each one of the variants found, it was tabulated its position, change of nucleotide, change of amino acid, and allele frequency. The allele frequency is the measure of the relative proportion of alleles in each population, expressed in percentage or in the unit. It is estimated by counting the number of times the allele is observed at a locus and dividing it by the total number of alleles studied.

The gene-allele frequency will be calculated by simple count:

$$
\mathrm{f}(\mathrm{A})=\text { \# alleles observed } / \text { \# total alleles }
$$

\section{Inclusion Criteria}

Exome results from patients with complex diseases that are different from MPS., obtained from the database of the 'Instituto de Genética Médica' - GENOMICS (Cali).

\section{Exclusion Criteria}

Exome results from patients who have not signed the respective informed consent - assent to sample collection and use of data.

\section{Bioethical Aspects}

- Protection of people and animals: The authors state that the procedures followed conformed to the ethical standards of responsible human experimentation committee and are aligned to the principles set out in the Declaration of Helsinki of the World Medical Association (WMA); the level of this research has been categorized as minimal risk since it presents a low risk of 
physical harm to the participant as it is a retrospective study of review results.

- Confidentiality of the data: The authors state that no patient data appear in this article and they have followed their work center's protocols.

- $\quad$ Right to privacy and informed consent: The authors declare that in this article there is no personal data of patients and the respective consent and informed assent was obtained for the processing of samples and use of data in a confidential manner by the patient's legal representative.

\section{Results}

When obtaining the results of the sequencing of complete exomes in 320 patients without a clinical diagnosis of MPS with suspicion of complex diseases, each one of the data was tabulated, noting the alleles observed for each locus concentrating the data to subsequently obtain the allele frequencies of each one of them. The data was organized according to the change of nucleotide, amino acid, and allele frequency in Table 1.

The alleles found in each one of the genes that compose the MPS complex presented different distributions of allele frequencies (Figure 1), which are detailed below.

Specifically, for the IDUA gene associated with MPS I, 121 alleles were identified of which the changes with the highest frequencies were p.His33Gln (0.32809), the $\mathrm{T}>\mathrm{C}$ transition located in position Chr4:983060 (0.31434), the $\mathrm{A}>\mathrm{G}$ transition located in position Chr4:985727 (0.28880), and the $\mathrm{C}>\mathrm{T}$ transition located in position Chr4:983809 (0.24558), all these alleles have been associated with benign phenotypes and are not reported in patients presenting the disease.

For the IDS gene related to MPS II, 12 alleles were identified. Among the most frequent for this group, it was reported the one found in the synonym mutation p.Thr146=, identified with a frequency of 0.10806 . Followed, the change $\mathrm{G}>\mathrm{GA}$ was in ChrX:14852574, with a frequency of 0.01768 . And the transitional change C $>\mathrm{A}$ was in ChrX:148586711, with a frequency of appearance of 0.01572 .

Concerning the genes associated with MPS III, 47 alleles associated with the SGSH gene were found. It is emphasized the presence of the change p.Val361Ile found in $163 / 320$ patients with a frequency of 0.32024 , the intronic variants $663+17 \mathrm{~T}>\mathrm{C}$ and 250-26C $>\mathrm{T}$ reported with a frequency of 0.29470 and 0.23576 respectively, and the synonym variant p.Arg820Trp that presented an allele frequency of 0.17682 . For the NAGLU gene, 36 allele variants were found; the synonym allele p.Ser141= showed a frequency of 0.36149 . Likewise, the alleles $531+50 \mathrm{G}>\mathrm{C}$, p.Arg737Gly, and the change T>C located in Chr17:40699332, presented frequencies $0.33399,0.34971$, and 0.35560 , respectively. In the HGSNAT gene, 27 allele variants were found. Of these, the ones that showed a higher frequency were p.tyr583 $=(0.36346)$ and the alleles, located in Chr8: 43033368, consistent with the

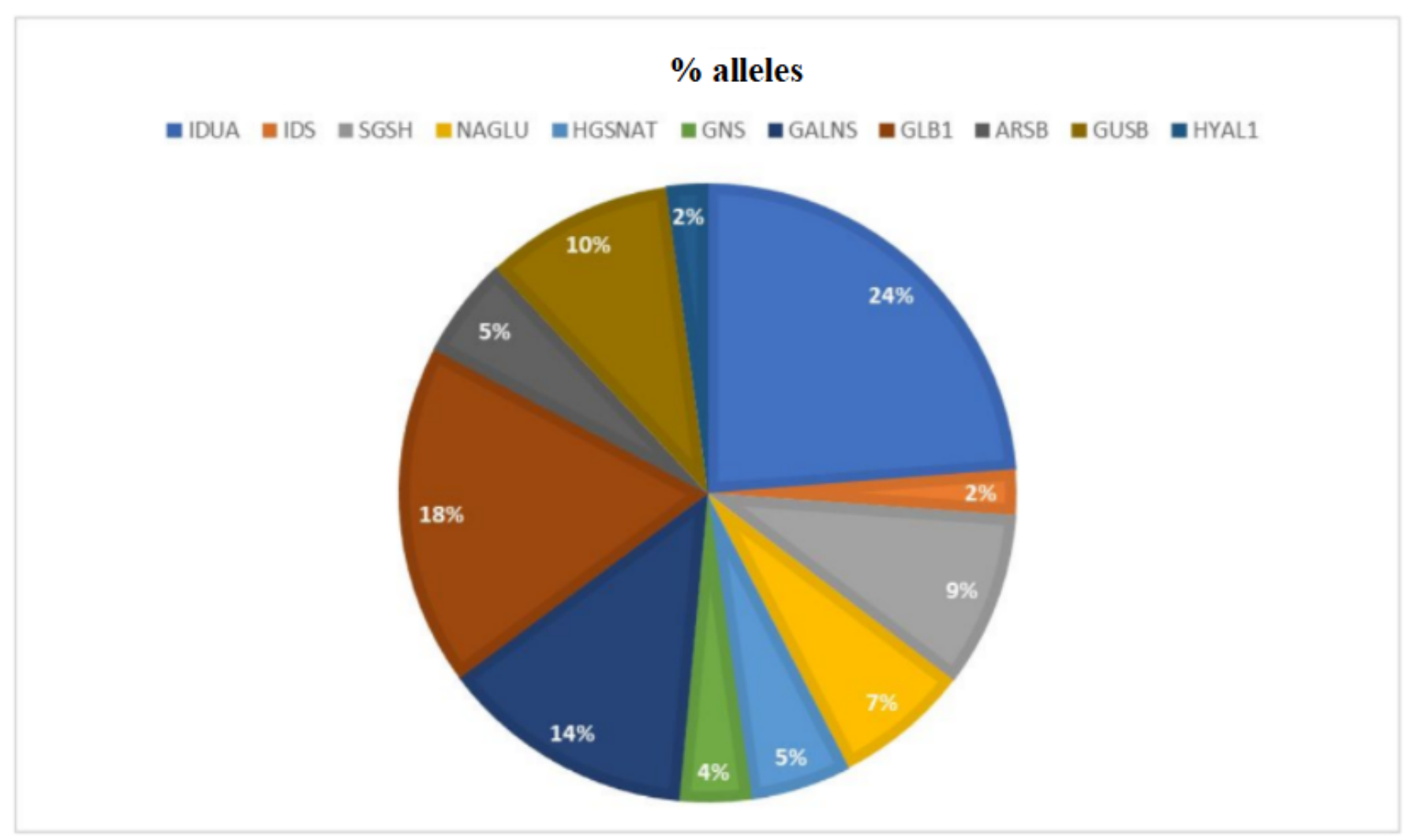

Figure 1. Percentage of alleles found in genes associated with the MPS complex. 
Table 1. Allelic frequency found in gene variants associated with the MPS complex.

\begin{tabular}{|c|c|c|c|c|c|}
\hline Type MPS & Gen & Position & nucleotide change & Amino acid change & Allelic frequency \\
\hline & IDUA & Chr4: 980664 & $\mathrm{C}>\mathrm{A}$ & & 0,01179 \\
\hline & IDUA & Chr4:980828 & $\mathrm{C}>\mathrm{A}$ & & 0,00393 \\
\hline & IDUA & Chr4:980841 & $\mathrm{C}>\mathrm{A}$ & & 0,00393 \\
\hline & IDUA & Chr4:980865 & $G>A$ & & 0,00393 \\
\hline & IDUA & Chr4:980883 & $11 \mathrm{~T}>\mathrm{C}$ & p.Leu4Pro & 0,00393 \\
\hline & IDUA & Chr4:980896 & $24 C>A$ & p.Ala8= & 0,21807 \\
\hline & IDUA & Chr4:980932 & $60 \mathrm{G}>\mathrm{A}$ & p.Ala20 = & 0,21415 \\
\hline & IDUA & Chr4:980971 & $99 \mathrm{~T}>\mathrm{G}$ & p.His33Gln & 0,32809 \\
\hline & IDUA & Chr4:980987 & c. $115 \mathrm{G}>\mathrm{A}$ & p.Ala39Thr & 0,00393 \\
\hline & IDUA & Chr4:981077 & $\mathrm{C}>\mathrm{A}$ & & 0,00393 \\
\hline & IDUA & Chr4:981546 & $\mathrm{C}>\mathrm{A}$ & & 0,00393 \\
\hline & IDUA & Chr4:981550 & $C>A$ & & 0,00393 \\
\hline & IDUA & Chr4:981564 & $\mathrm{C}>\mathrm{A}$ & & 0,00393 \\
\hline & IDUA & Chr4:981597 & $\mathrm{C}>\mathrm{A}$ & & 0,00393 \\
\hline & IDUA & Chr4:981673 & $235 G>A$ & p.Ala79Thr & 0,00393 \\
\hline & IDUA & Chr4:981684 & $246 C>G$ & p.His82Gln & 0,00393 \\
\hline & IDUA & Chr4:981746 & $\mathrm{C}>\mathrm{A}$ & & 0,00393 \\
\hline & IDUA & Chr4:981752 & $\mathrm{G}>\mathrm{A}$ & & 0,00393 \\
\hline & IDUA & Chr4:981786 & $\mathrm{C}>\mathrm{A}$ & & 0,00393 \\
\hline & IDUA & Chr4:982635 & $\mathrm{C}>\mathrm{A}$ & & 0,00982 \\
\hline & IDUA & Chr4:982736 & $C>G$ & & 0,00393 \\
\hline & IDUA & Chr4:982756 & $\mathrm{C}>\mathrm{A}$ & & 0,00393 \\
\hline \multirow{24}{*}{$\begin{array}{l}\text { MPS I - Hurler } \\
\text { syndrome }\end{array}$} & IDUA & Chr4:982784 & $\mathrm{C}>\mathrm{A}$ & & 0,00393 \\
\hline & IDUA & Chr4:982812 & $\mathrm{C}>\mathrm{A}$ & & 0,00393 \\
\hline & IDUA & Chr4:982814 & $\mathrm{C}>\mathrm{A}$ & & 0,00393 \\
\hline & IDUA & Chr4:982852 & $\mathrm{G}>\mathrm{A}$ & & 0,21415 \\
\hline & IDUA & Chr4:982913 & $\mathrm{G}>\mathrm{C}$ & & 0,00393 \\
\hline & IDUA & Chr4:982985 & $\mathrm{C}>\mathrm{A}$ & & 0,00393 \\
\hline & IDUA & Chr4:983014 & $\mathrm{G}>\mathrm{C}$ & & 0,00982 \\
\hline & IDUA & Chr4:983060 & $\mathrm{T}>\mathrm{C}$ & & 0,31434 \\
\hline & IDUA & Chr4:983130 & $G>T$ & & 0,00393 \\
\hline & IDUA & Chr4:983139 & $\mathrm{C}>\mathrm{A}$ & & 0,00393 \\
\hline & IDUA & Chr4:983178 & $1549 G>A$ & p.Gly517Arg & 0,00393 \\
\hline & IDUA & Chr4:983183 & $C>T$ & & 0,00393 \\
\hline & IDUA & Chr4:983197 & $\mathrm{G}>\mathrm{A}$ & & 0,00393 \\
\hline & IDUA & Chr4:983216 & $C>T$ & & 0,00196 \\
\hline & IDUA & Chr4:983254 & $\mathrm{C}>\mathrm{A}$ & & 0,00393 \\
\hline & IDUA & Chr4:983280 & $\mathrm{G}>\mathrm{A}$ & & 0,00393 \\
\hline & IDUA & Chr4:983296 & $\mathrm{G}>\mathrm{A}$ & & 0,00393 \\
\hline & IDUA & Chr4:983314 & $C>T$ & & 0,00786 \\
\hline & IDUA & Chr4:983316 & $\mathrm{C}>\mathrm{A}$ & & 0,00393 \\
\hline & IDUA & Chr4:983323 & $\mathrm{C}>\mathrm{A}$ & & 0,00393 \\
\hline & IDUA & Chr4:983331 & $\mathrm{T}>\mathrm{C}$ & & 0,00393 \\
\hline & IDUA & Chr4:983342 & $C>T$ & & 0,00393 \\
\hline & IDUA & Chr4:983550 & $A>G$ & & 0,00393 \\
\hline & IDUA & Chr4:983574 & $C>T$ & & 0,00393 \\
\hline
\end{tabular}


Table 1. Cont..

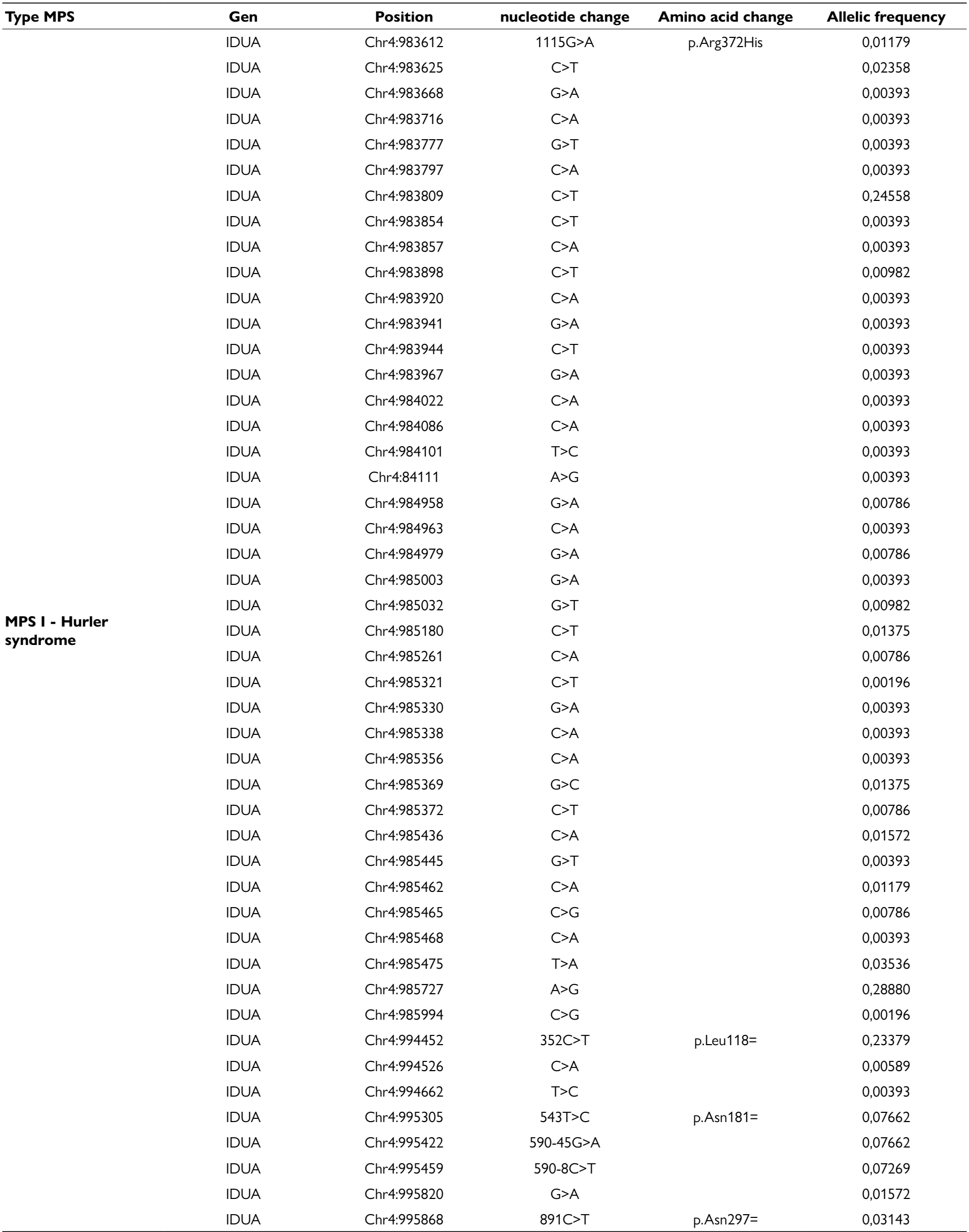


Table 1. Cont..

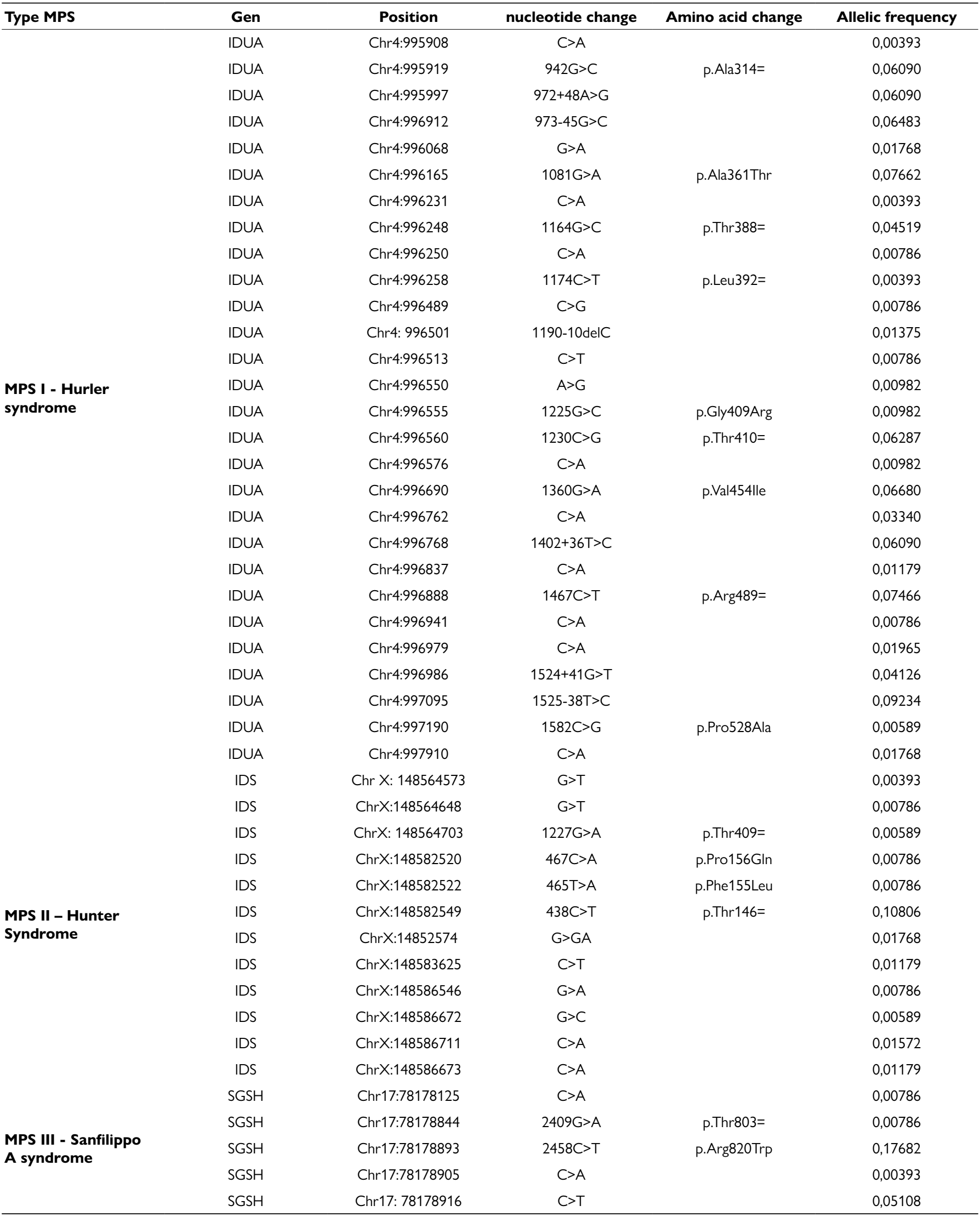


Table 1. Cont.

\begin{tabular}{|c|c|c|c|c|c|}
\hline Type MPS & Gen & Position & nucleotide change & Amino acid change & Allelic frequency \\
\hline & SGSH & Chr17:78178931 & $2496 C>T$ & pl.Leu832= & 0,00393 \\
\hline & SGSH & Chr17:78178998 & $C>A$ & & 0,00786 \\
\hline & SGSH & Chr17:78180817 & $C>A$ & & 0,00196 \\
\hline & SGSH & Chr17:78180840 & $2763 C>T$ & p.lle921= & 0,00982 \\
\hline & SGSH & Chr17:78181957 & $\mathrm{G}>\mathrm{A}$ & & 0,01179 \\
\hline & SGSH & Chr17:78181958 & $2829 \mathrm{C}>\mathrm{T}$ & p.Gly943= & 0,00786 \\
\hline & SGSH & Chr17:78181996 & $A>G$ & & 0,01375 \\
\hline & SGSH & Chr17:78182014 & $2885 G>A$ & p.Arg962Gln & 0,00589 \\
\hline & SGSH & Chr17:78182125 & $\mathrm{C}>\mathrm{A}$ & & 0,03143 \\
\hline & SGSH & Chr17:78184314 & $\mathrm{G}>\mathrm{A}$ & & 0,00393 \\
\hline & SGSH & Chr17:78184374 & $C>A$ & & 0,00393 \\
\hline & SGSH & Chr17:78184679 & $1081 \mathrm{G}>\mathrm{A}$ & p.Val361lle & 0,32024 \\
\hline & SGSH & Chr17:78184702 & $\mathrm{T}>\mathrm{C}$ & & 0,00393 \\
\hline & SGSH & Chr17:78184711 & $\mathrm{G}>\mathrm{A}$ & & 0,00589 \\
\hline & SGSH & Chr17.78184713 & $\mathrm{C}>\mathrm{A}$ & & 0,00393 \\
\hline & SGSH & Chr17:78184761 & $\mathrm{G}>\mathrm{A}$ & & 0,00589 \\
\hline & SGSH & Chr17:78185953 & $\mathrm{C}>\mathrm{A}$ & & 0,00786 \\
\hline & SGSH & Chr17:78186077 & $746-4 A>G$ & & 0,00982 \\
\hline & SGSH & Chr17:78187565 & $\mathrm{C}>\mathrm{T}$ & & 0,00589 \\
\hline & SGSH & Chr17:78187673 & $675 \mathrm{C}>\mathrm{T}$ & p.Phe225= & 0,00786 \\
\hline \multirow{24}{*}{$\begin{array}{l}\text { MPS III - Sanfilippo } \\
\text { A syndrome }\end{array}$} & SGSH & Chr17: 78187720 & $664-36 \mathrm{~T}>\mathrm{C}$ & & 0,16503 \\
\hline & SGSH & Chr17:78187721 & 664-39_664-38delCT & & 0,12181 \\
\hline & SGSH & Ch17:78187734 & $664-50 \mathrm{G}>\mathrm{A}$ & & 0,02161 \\
\hline & SGSH & Chr17:78187884 & $\mathrm{T}>\mathrm{C}$ & & 0,00786 \\
\hline & SGSH & Chr17:78187954 & $663+17 T>C$ & & 0,29470 \\
\hline & SGSH & Chr17:78187993 & $\mathrm{G}>\mathrm{T}$ & & 0,00589 \\
\hline & SGSH & Ch17:78188028 & $G>A$ & & 0,00589 \\
\hline & SGSH & Chr17:78188794 & $C>T$ & & 0,01179 \\
\hline & SGSH & Chr17:78188963 & $250-26 C>T$ & & 0,23576 \\
\hline & SGSH & Chr17:78190794 & $C>A$ & & 0,00589 \\
\hline & SGSH & Chr17:78191017 & $G>T$ & & 0,00786 \\
\hline & SGSH & Chr17:78191030 & $89-39 G>A$ & & 0,03733 \\
\hline & SGSH & Chr17:78191036 & $89-45 G>A$ & & 0,03733 \\
\hline & SGSH & Chr17:78193986 & $\mathrm{C}>\mathrm{A}$ & & 0,02358 \\
\hline & SGSH & Chr17:78194002 & $88+23 C>G$ & & 0,00589 \\
\hline & SGSH & Chr17:78196622 & $C>A$ & & 0,00393 \\
\hline & SGSH & Chr17:78196612 & $C>A$ & & 0,00393 \\
\hline & SGSH & Chr17:78196506 & $C>A$ & & 0,00393 \\
\hline & SGSH & Chr17:78195371 & $G>T$ & & 0,00393 \\
\hline & SGSH & Chr17:78195366 & $\mathrm{T}>\mathrm{G}$ & & 0,00393 \\
\hline & SGSH & Chr17:78194160 & $G>A$ & & 0,00589 \\
\hline & SGSH & Chr17:78194084 & $\mathrm{G}>\mathrm{C}$ & & 0,00393 \\
\hline & NAGLU & Chr17:40688275 & $C>A$ & & 0,00393 \\
\hline & NAGLU & Chr17:40688327 & $\mathrm{C}>\mathrm{A}$ & & 0,01179 \\
\hline \multirow{3}{*}{$\begin{array}{l}\text { MPS III - Sanfilippo } \\
\text { B syndrome }\end{array}$} & NAGLU & Chr17:40688359 & $C>A$ & & 0,01375 \\
\hline & NAGLU & Chr17:40688395 & $C>A$ & & 0,00589 \\
\hline & NAGLU & Chr17:40688426 & $\mathrm{G}>\mathrm{T}$ & & 0,00393 \\
\hline
\end{tabular}


Table 1. Cont.

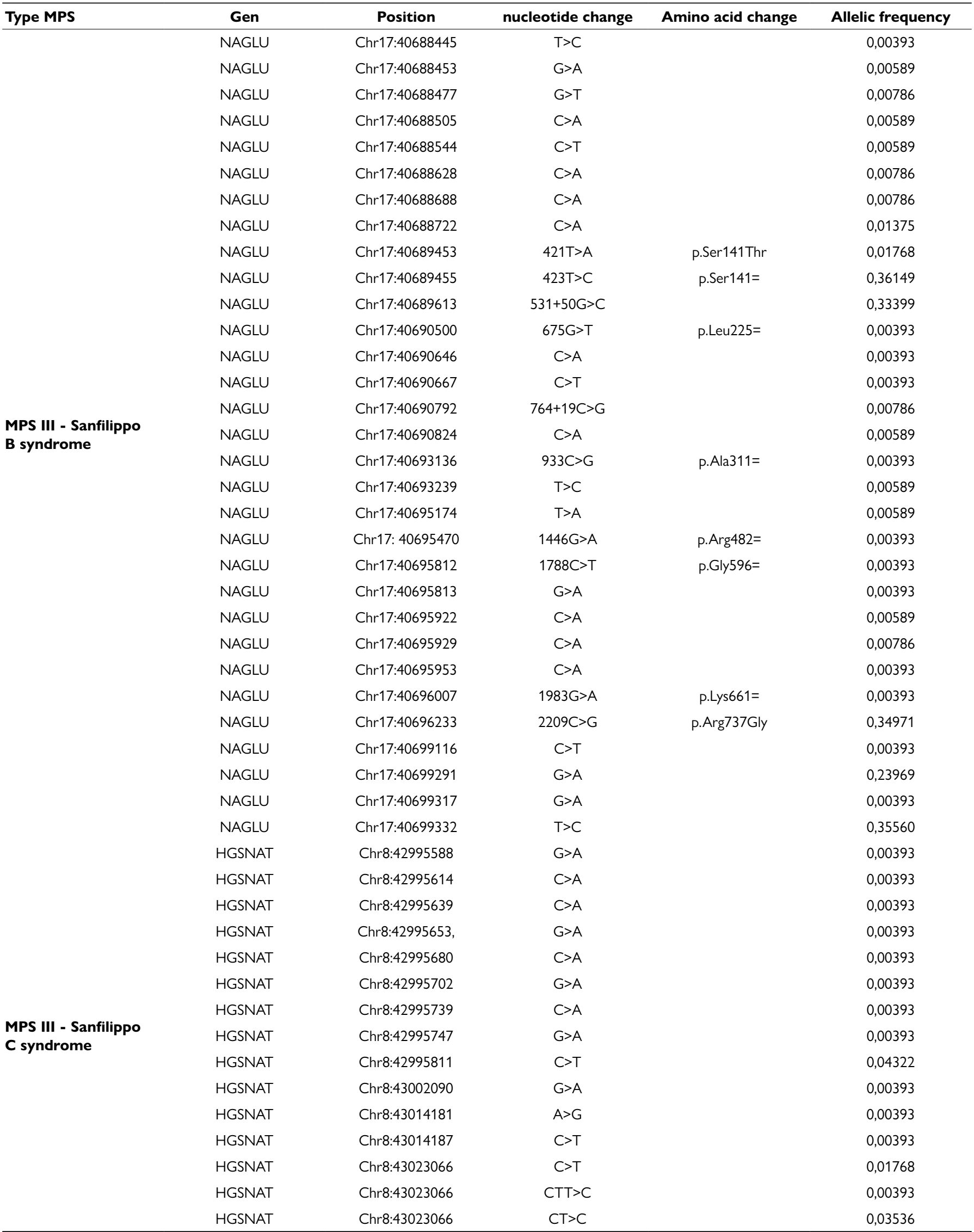


Table 1. Cont..

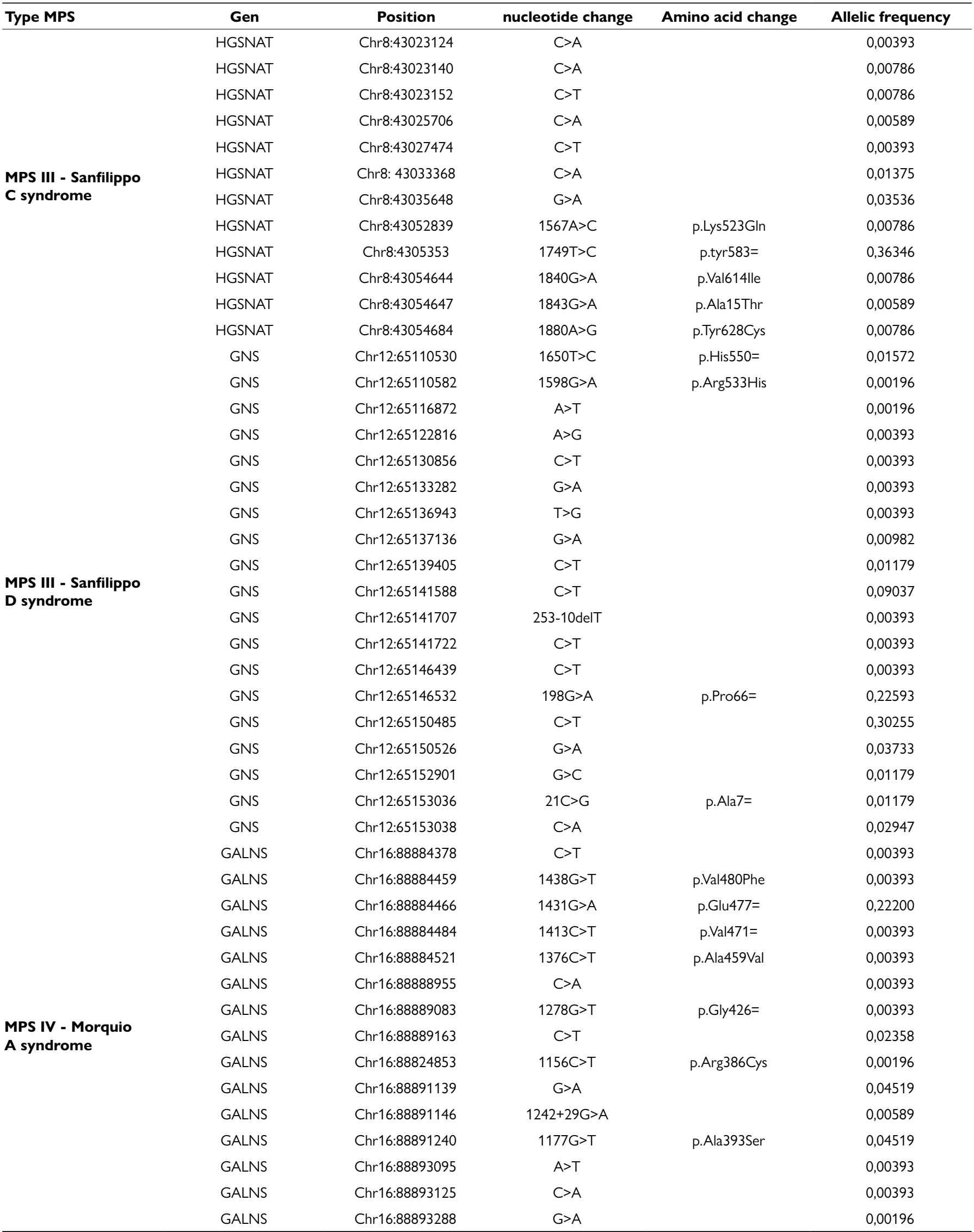


Table 1. Cont..

\begin{tabular}{|c|c|c|c|c|c|}
\hline Type MPS & Gen & Position & nucleotide change & Amino acid change & Allelic frequency \\
\hline \multirow{46}{*}{$\begin{array}{l}\text { MPS IV - Morquio } \\
\text { A syndrome }\end{array}$} & GALNS & Chr16:88893295 & $T>C$ & & 0,00786 \\
\hline & GALNS & Chr16:88898507 & $901 \mathrm{G}>\mathrm{T}$ & p.Gly301Cys & 0,00393 \\
\hline & GALNS & Chr16:88901579 & $898+42 G>C$ & & 0,03733 \\
\hline & GALNS & Chr16:88901594 & $C>T$ & & 0,00393 \\
\hline & GALNS & Chr16:88901596 & $898+25 C>G$ & & 0,03733 \\
\hline & GALNS & Chr16:88901673 & $846 C>T$ & p.Phe282= & 0,04126 \\
\hline & GALNS & Chr16:88902111 & $758+22 C>T$ & & 0,03733 \\
\hline & GALNS & Chr16:88902183 & $708 \mathrm{C}>\mathrm{T}$ & p.His $236=$ & 0,15717 \\
\hline & GALNS & Chr16:88902199 & $692 C>G$ & p.Ala231Gly & 0,03733 \\
\hline & GALNS & Chr16:88902276 & $634-19 G>A$ & & 0,15914 \\
\hline & GALNS & Chr16:88902277 & $634-20 C>T$ & & 0,07662 \\
\hline & GALNS & Chr16:88902643 & $599 \mathrm{C}>\mathrm{T}$ & p.Thr200Met & 0,01572 \\
\hline & GALNS & Chr16:88903999 & $C>T$ & & 0,00393 \\
\hline & GALNS & Chr16:88904019 & $G>A$ & & 0,00393 \\
\hline & GALNS & Chr16:88904020 & $566+10 C>T$ & & 0,00393 \\
\hline & GALNS & Chr16:88904086 & $510 \mathrm{~T}>\mathrm{C}$ & p.Tyr170= & 0,04126 \\
\hline & GALNS & Chr16:88904166 & $C>A$ & & 0,00393 \\
\hline & GALNS & Chr16:88904210 & $C>A$ & & 0,00393 \\
\hline & GALNS & Chr16:88904220 & $C>A$ & & 0,00393 \\
\hline & GALNS & Chr16:88904222 & $C>A$ & & 0,00393 \\
\hline & GALNS & Chr16:88907499 & $T>C$ & & 0,00393 \\
\hline & GALNS & Chr16:88907516 & $G>A$ & & 0,00393 \\
\hline & GALNS & Chr16:88907553 & $C>A$ & & 0,00393 \\
\hline & GALNS & Chr16:88908306 & $318 \mathrm{C}>\mathrm{T}$ & p.Asn106= & 0,01572 \\
\hline & GALNS & Chr16:88908418 & $G>A$ & & 0,00393 \\
\hline & GALNS & Chr16:88909065 & c. $244+49 C>T$ & & 0,00982 \\
\hline & GALNS & Chr16:88909081 & $C>A$ & & 0,00393 \\
\hline & GALNS & Chr16:88909086 & $C>T$ & & 0,00393 \\
\hline & GALNS & Chr16:88909095 & $244+19 C>T$ & & 0,07859 \\
\hline & GALNS & Chr16:88909118 & $240 G>A$ & p.Ser80 $=$ & 0,00393 \\
\hline & GALNS & Chr16:88909159 & $199 C>A$ & p.Leu67Met & 0,02358 \\
\hline & GALNS & Chr16:88909161 & $C>T$ & & 0,00393 \\
\hline & GALNS & Chr16:88909445 & $T>C$ & & 0,01375 \\
\hline & GALNS & Chr16:88909446 & $C>T$ & & 0,00393 \\
\hline & GALNS & Chr16:88909494 & $T>G$ & & 0,00393 \\
\hline & GALNS & Chr16:88921634 & $G>G$ & & 0,17092 \\
\hline & GALNS & Chr16:88921925 & $C>A$ & & 0,00393 \\
\hline & GALNS & Chr16:88922603 & $G>A$ & & 0,00786 \\
\hline & GALNS & Chr16:88922630 & $T>C$ & & 0,01179 \\
\hline & GALNS & Chr16:88923157 & $C>A$ & & 0,00786 \\
\hline & GALNS & Chr16:88923377 & $-92 \mathrm{~T}>\mathrm{C}$ & & 0,00982 \\
\hline & GALNS & Chr16:88923391 & $C>A$ & & 0,03340 \\
\hline & GALNS & Chr16:88923505 & $C>A$ & & 0,00393 \\
\hline & GALNS & Chr16:88923669 & $\mathrm{T}>\mathrm{C}$ & & 0,00393 \\
\hline & GALNS & Chr16:88925112 & $A>G$ & & 0,00393 \\
\hline & GALNS & Chr16:88926089 & $A>G$ & & 0,04715 \\
\hline
\end{tabular}


Table 1. Cont.

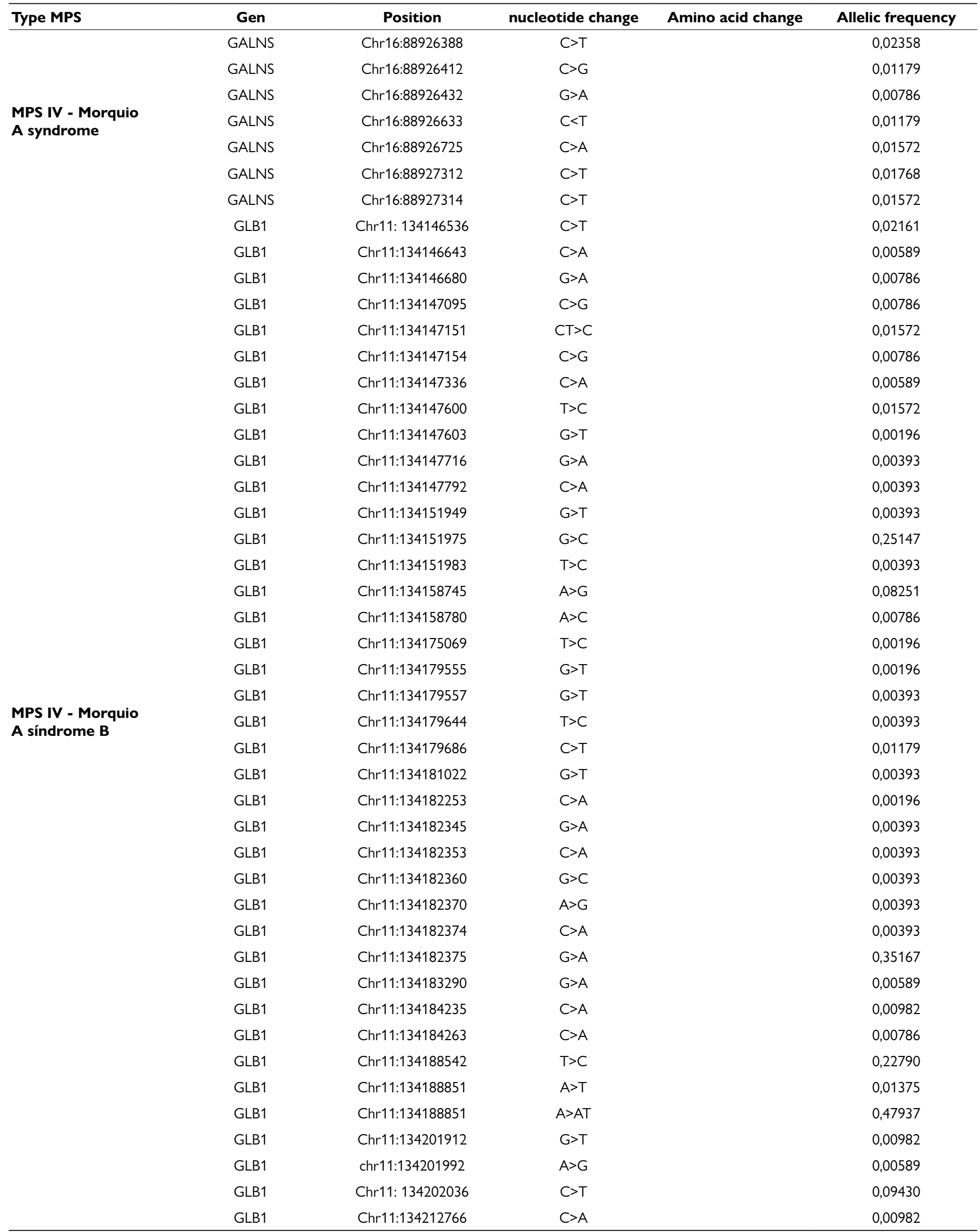


Table 1. Cont..

\begin{tabular}{|c|c|c|c|c|c|}
\hline Type MPS & Gen & Position & nucleotide change & Amino acid change & Allelic frequency \\
\hline & GLB1 & Chr11:134212821 & $C>A$ & & 0,00982 \\
\hline & GLB1 & Chr11:134212829 & $T>C$ & & 0,00393 \\
\hline & GLB1 & Chr11:134214326 & $C>G$ & & 0,01179 \\
\hline & GLB1 & Chr11:134226201 & $C>T$ & & 0,00393 \\
\hline & GLB1 & Chr11:134226244 & $G>A$ & & 0,00393 \\
\hline & GLB1 & Chr11:134226278 & $C>T$ & & 0,04322 \\
\hline & GLB1 & Chr11:134226291 & $A>G$ & & 0,02947 \\
\hline & GLB1 & Chr11:134229010 & $G>A$ & & 0,00786 \\
\hline & GLB1 & Chr11:134229016 & $C>A$ & & 0,00589 \\
\hline & GLB1 & Chr11:134234269 & $A>G$ & & 0,00786 \\
\hline & GLB1 & Chr11:134237113 & $C>A$ & & 0,19253 \\
\hline & GLB1 & Chr11:134237116 & $\mathrm{G}>\mathrm{C}$ & & 0,00393 \\
\hline & GLB1 & Chr11:134237192 & $G>T$ & & 0,00393 \\
\hline & GLB1 & Chr11:134238569 & $C>T$ & & 0,00393 \\
\hline & GLB1 & Chr11:134238599 & $C>T$ & & 0,01179 \\
\hline & GLB1 & Chr11:134238603 & $G>A$ & & 0,03340 \\
\hline & GLB1 & Chr11:134239760 & $C>T$ & & 0,00786 \\
\hline & GLB1 & Chr11:134240213 & $C>T$ & & 0,00393 \\
\hline & GLB1 & Chr11:134240949 & $T>C$ & & 0,01768 \\
\hline & GLB1 & Chr11:134241248 & $C>A$ & & 0,00589 \\
\hline & GLB1 & Chr3:33055688 & $1594 A>G$ & p.Ser532Gly & 0,01768 \\
\hline & GLB1 & Chr3:33055705 & $1577 G>A$ & p.Gly526Asp & 0,00393 \\
\hline \multirow{24}{*}{$\begin{array}{l}\text { MPS IV - Morquio } \\
\text { A síndrome B }\end{array}$} & GLB1 & Chr3:33055721 & $1561 C>T$ & p.Arg521Cys & 0,36149 \\
\hline & GLB1 & Chr3:33055849 & $A>G$ & & 0,00393 \\
\hline & GLB1 & Chr3:33058254 & $1426 \mathrm{C}>\mathrm{T}$ & p.Leu476= & 0,00196 \\
\hline & GLB1 & Chr3:33058304 & $T>C$ & & 0,00393 \\
\hline & GLB1 & Chr3:33058310 & 1370G>A & p.Arg457Gln & 0,00393 \\
\hline & GLB1 & Chr3:33058314 & $C>A$ & & 0,00589 \\
\hline & GLB1 & Chr3:33059981 & $913 \mathrm{C}>\mathrm{T}$ & p.Leu305Phe & 0,00589 \\
\hline & GLB1 & Chr3:rs34421970 & $T>C$ & & 0,00982 \\
\hline & GLB1 & Chr3: 33063050 & $1233+8 \mathrm{~T}>\mathrm{C}$ & & 0,11198 \\
\hline & GLB1 & Chr3:33093515 & $793-19 C>T$ & & 0,00786 \\
\hline & GLB1 & Chr3:33094973 & $792+10 G>T$ & & 0,04912 \\
\hline & GLB1 & Chr3:33106934 & $552+21 \mathrm{G}>\mathrm{A}$ & & 0,00589 \\
\hline & GLB1 & Chr3:33107060 & $458-11 T>C$ & & 0,02947 \\
\hline & GLB1 & Chr3:33109814 & $C>A$ & & 0,00196 \\
\hline & GLB1 & Chr3:33110383 & $325 C>T$ & p.Arg109Trp & 0,01375 \\
\hline & GLB1 & Chr3:33113992 & $T>G$ & & 0,01768 \\
\hline & GLB1 & Chr3:33118597 & $G>T$ & & 0,00393 \\
\hline & GLB1 & Chr3:33118631, & $G>A$ & & 0,05894 \\
\hline & GLB1 & Chr3:33118725 & $C>T$ & & 0,00393 \\
\hline & GLB1 & Chr3:33134385 & $G>A$ & & 0,00982 \\
\hline & GLB1 & Chr3:33134392 & $\mathrm{G}>\mathrm{A}$ & & 0,00589 \\
\hline & GLB1 & Chr3:33134598 & $T>C$ & & 0,09430 \\
\hline & GLB1 & Chr3:33134676 & $C>A$ & & 0,00786 \\
\hline & GLB1 & Chr3:33134707 & GTCC>G & & 0,00393 \\
\hline
\end{tabular}


Table 1. Cont..

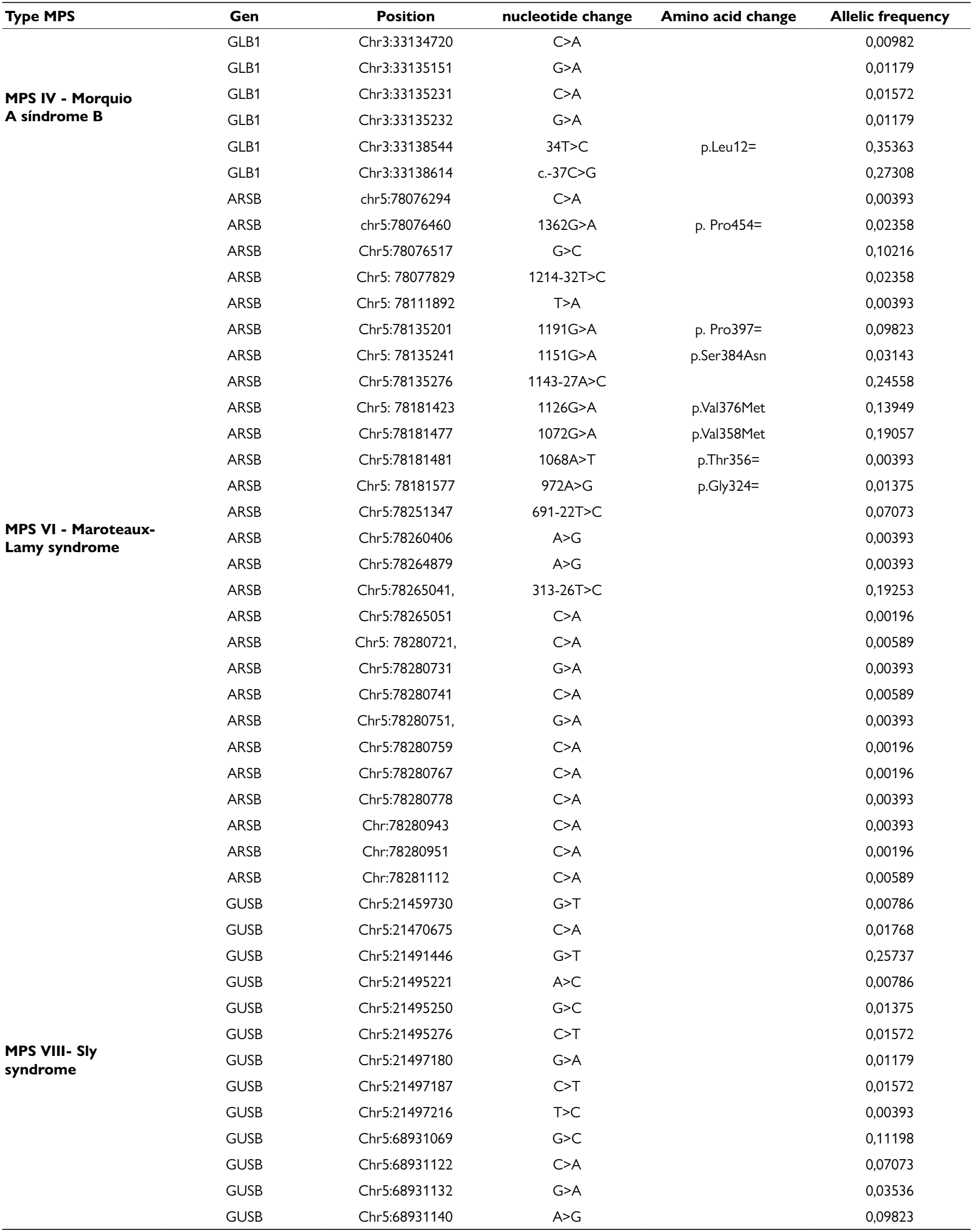


Table 1. Cont..

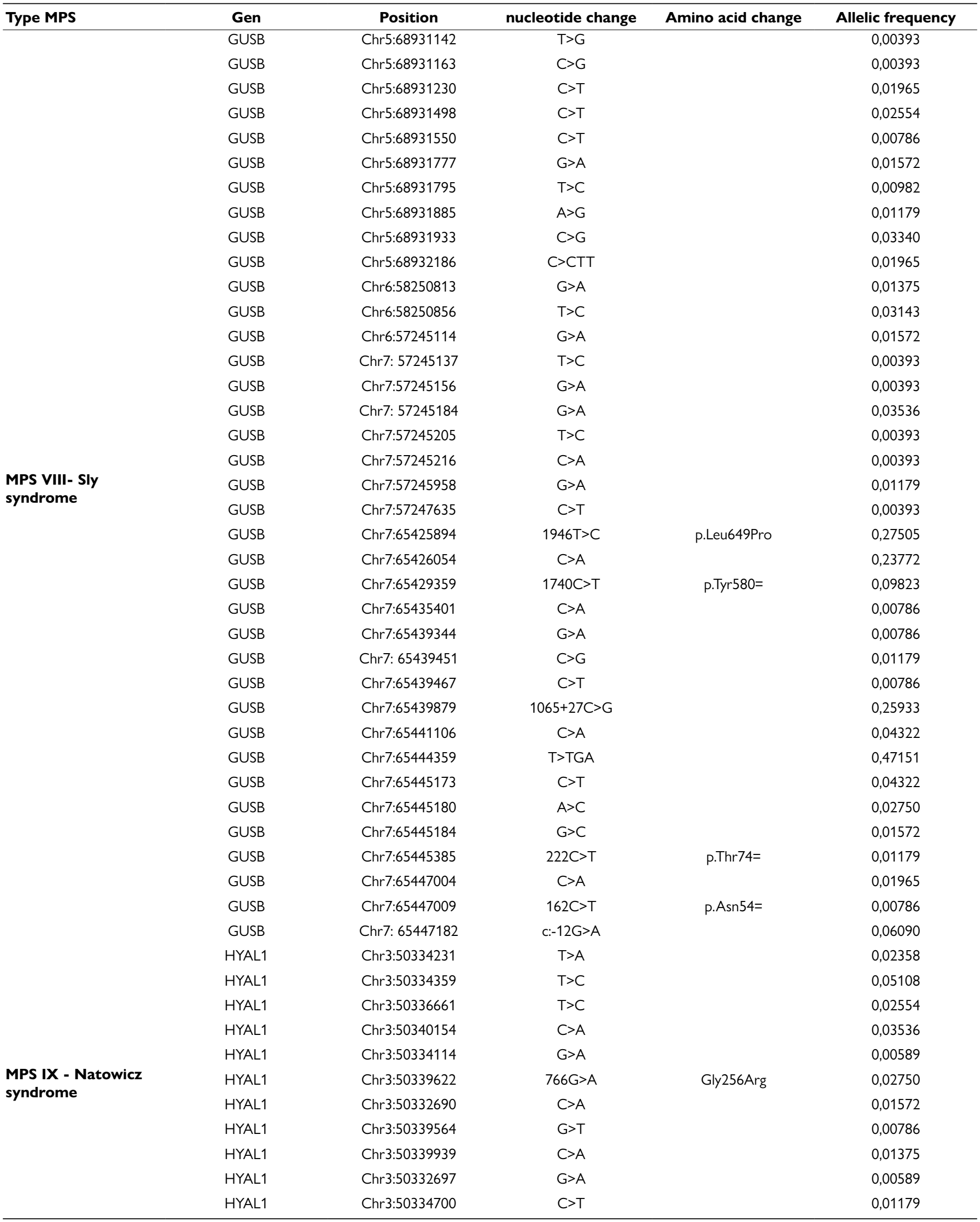


transversion $\mathrm{C}>\mathrm{A}(0.01375)$ and $\mathrm{Chr} 8: 42995811 \mathrm{C}>\mathrm{T}$ (0.04322). As for the 19 alleles reported in the GNS gene, the one with the highest frequency of appearance was the new report occurred in the position Chr12:65150485 C>T (0.30255); followed by the allele p.Pro66 $=$ with a frequency of 0.22593 and the change $\mathrm{C}>\mathrm{T}$ occurred in the position Chr12:65141588 (0.09037).

Regarding those associated with MPS IV, 68 different alleles were reported for the GALNS gene, having the variants p.Glu477=, 634-19G >A, p.His236= frequencies of 0.22200, 0.15914 , and 0.15717 , respectively. Likewise, for the gene GLB1, 91 allelic variants were reported, of which the allele p.Leu12= was presented with the highest frequency (0.35363), followed by p.Arg521Cys (0.36149) and c.-37C > G (0.27308).

For the MPS VI associated ARSB gene, 27 allelic variants were tabulated. The intronic change 1143-27A $>\mathrm{C}$ showed higher frequency $(0.24558)$, followed by $313-26 \mathrm{~T}>\mathrm{C}(0.19253)$ and p.Val358Met (0.19057).

Concerning the GUSB gene associated with MPSVIII, 50 allelic variants were found. The change located in Chr7:65444359, consistent with T>TGA showed the highest frequency among all the findings of the work, showing allele frequency values of 0.47151 with presence in $240 / 320$ samples.

Finally, over the 11 allele variants found in the gene HYAL1, associated with MPS IX, it was reported that the change Chr3:50334359 $\mathrm{T}>\mathrm{C}$ was the one that showed the highest frequency $(0.05108)$ among all the variants of this gene, followed by Gly256Arg (0.02750).

\section{Discussion}

In recent years, with the advent of diagnostic techniques based on massive DNA sequencing, the increase of information regarding variants associated with different diseases has been made possible and facilitated. This technology has allowed the identification of thousands of alleles relate to the etiopathogenesis of a wide variety of diseases, as well as the recognition of biomarkers that could be used to inform disease prediction, identification of causal mechanisms, and the prioritization of new biological targets in drug discovery programs [11].

There are very few studies and with limited information on the calculation of allele frequency estimates of the variants of the MPS complex in the Colombian population, geographically delimited, with a reduced number of participants, and with technical methodological deficiencies, which leads to a partial and not adequate knowledge for its integrated approach that includes a family study and identification of carriers for the recognition of the risk of heritability.

For the present study of allele frequency carried out through the complete exome sequencing using the Illumina platform in 320 patients from South-Western Colombia without a clinical diagnosis of MPS, 509 variants associated with the MPS complex were reported. Among them, 262 had not been previously reported, which enhances the importance of their study, classification, effect in the functionality of the protein, and population expression.

The allele frequencies reported in these patients with diverse pathologies and not clinically diagnosed with MPS ranged from 0.00393 ( 2 alleles) to 0.47937 (248 alleles). Research conducted by Gomez et al. (2012) on patients affected by MPS, for MPS I and II, found 0.45 cases per 100,000 births for each of them. The frequency found for "MPS III in Cundinamarca and Boyacá, 0.17 cases per 100,000 live births. They did not find any patients with MPS type VII and IX [6].

The IDUA gene had the highest percentage of recognized alleles (24\%), 11 of which were distributed between exons 1 and 10 and introns 5 and 7 and found in the study by Bertola et al. (2011) with patients confirmed with MPS I [12]. The heterogeneity and the prevalence of mutations highlight the importance of multinational screening studies to help elucidate the genotype-phenotype relationship in disorders such as MPS I that is characterized by extensive allelic heterogeneity.

As for the alleles, $14 \%$ of them were identified in the GALNS gene, characterized by being one of the most studied worldwide, with high mutational frequencies. In this study, the variant c. $1431 \mathrm{G}>\mathrm{A}$ was the most frequent with $60 \%$ of the alleles, followed in frequency by the benign mutations p.H36= and the intronic variant $634-19 \mathrm{G}>\mathrm{A}$ with $41 \%$ and $34 \%$, respectively. These alleles were not present in the population affected by MPS IVA, and for that, they are considered polymorphisms [13, 14].

In all the other genes associated with the MPS complex, there were not found alleles that show a high incidence in the affected population. For this reason, the current literature about the possible mutations associated with these genes is reduced, generating a relevant component in the knowledge of the polymorphisms found in people without the disease.

The frequency of the genetic variants varies by changing the allele frequencies. Thus, if there are two alleles in the same gene, it is polymorphic, and each new allele that emerges in the population increases the polymorphism of the gene. To be considered as such, the frequency of the most common allele must be less than 99\%, and for a rare allele, it must exceed at least $0.005 \%$ of the frequency in the population; alleles that do not reach these frequencies are considered rare [15]. Genetic variants being aside from the scope of association studies with most statistical power is believed to contribute to a lack heritability of many human features, including common variants (denoted by a frequency of minor alleles $[\mathrm{MAF}]>5 \%$ ) of weak effect, low frequency (MAF 1-5\%), and rare variants (MAF $<1 \%$ ) of small to moderate effect, or a combination of both with several possible scenarios, all considered plausible in simulation studies $[16,17]$.

In conclusion, despite finding a great diversity of alleles in the genes associated with mucopolysaccharidosis, the vast majority of these have been associated with benign phenotypes and are not reported in patients presenting the disease, so they could be could be polymorphisms in this population without the disease

Additional efforts to discover associations driven by rare and low-frequency variants through exome sequencing and 
efforts in bioinformatics research allow continuous advances in the proportion of heritability explained by variants across the frequency spectrum. Heritability estimates for the entire genome depend, to a great extent, on assumptions about the imbalance of binding, frequency of the alleles, and genotype certainty. Therefore, future studies around these assumptions will be necessary to understand the impact of the alleles on the phenotype-genotype of patients.

Finally, studies based on allelic frequency estimates allow a better approach to the timely diagnosis and proper management of pathologies, since, by suggesting corresponding correlation tests of genotype and phenotypic indicators, to know the alleles present will allow to determine in a timelier manner the incidence and participation of many of these in the MPS complex.

\section{Funding}

The authors received no financial support for the research, authorship, and/or publication of this article.

\section{Declaration of Conflicting Interests}

The authors declare no conflict of interest.

\section{References}

1. Lin H-Y, Chan W-C, Chen L-J, et al. Ophthalmologic manifestations in Taiwanese patients with mucopo lysaccharidoses. Mol Genet Genomic Med. 2019;7(5):e00617. doi:10.1002/mgg3.617

2. Moreno-Giraldo LJ, Satizábal-Soto JM, Sanchéz-Gomez A. Genomic variability of the mucopolysaccharidosis complex in southwestern Colombia. Genet Mol Res. 2020;19(2):GMR18502. doi:10.4238/gmr 18502

3. Manger B. Lysosomal storage diseases. $Z$ Rheumatol. 2010;69(6):527-538. doi:10.1007/s00393-010-0627-z

4. Stapleton M, Hoshina H, Sawamoto K, et al. Critical review of current MPS guidelines and management. Mol Genet Metab. 2019;126(3):238-245. doi:10.1016/j. ymgme.2018.07.001

5. Khan SA, Peracha H, Ballhausen D, et al. Epidemiology of mucopolysaccharidoses. Mol Genet Metab. 2017;121(3):227240. doi:10.1016/j.ymgme.2017.05.016

6. Gómez AM, García-Robles R, Suárez-Obando F. Estimación de las frecuencias de las mucopolisacaridosis y análisis de agrupamiento espacial en los departamentos de Cundinamarca y Boyacá. Biomedica. 2012;32(4):602609. doi:10.1590/S0120-41572012000400015
7. Ministerio de Salud y Protección Social. Resolución número 005265 de 2018. Bogota(CO): Ministerio de Salud; 2018. http://www.acgh.com.co/publicaciones/149-reporteenfermedades-huerfanas-raras-sivigila. Accessed 12 July 2019.

8. Instituto Nacional de Salud. Comportamiento epidemiológico de las enfermedades huérfanas. Colombia, 2016 hasta semana epidemiológica 05 de 2019. Bogota(CO): Instituto Nacional de Salud; 2019.

9. Barrera LA. Estudios Bioquímicos de los errores innatos del metabolismo en Colombia, durante dos décadas. Rev Acad Colomb Cienc. 2009;33:377-394.

10. Bernal JE, Briceno I. Genetic and other diseases in the pottery of Tumaco-La Tolita culture in Colombia-Ecuador. Clin Genet. 2006;70(3):188-191. doi:10.1111/j.1399-0004. 2006.00670.x

11. Amorim CEG, Gao Z, Baker Z, et al. The population genetics of human disease: The case of recessive, lethal mutations. PLoS Genet. 2017;13(9):e1006915. doi: 10.1371/ journal.pgen.1006915

12. Bertola F, Filocamo M, Casati G, et al. IDUA mutational profiling of a cohort of 102 European patients with mucopolysaccharidosis type I: identification and characterization of 35 novel $\alpha$-L-iduronidase (IDUA) alleles. Hum Mutat. 2011;32(6):e2189-e2210. doi:10.1002/ humu. 21479

13. Cacioti A, Tonin R, Rigoldi M, et al. Optimizing the molecular diagnosis of GALNS: novel methods to define and characterize Morquio-A syndrome-associated mutations. Hum Mutat. 2015;36(3):357-368. doi:10.1002/humu.22751

14. Morrone A, Caciotti A, Atwood R, et al. Morquio a syndrome-associated mutations: A review of alterations in the GALNS gene and a new locus-specific database. Hum Mutat. 2014;35(11):1271-1279. doi: 10.1002/humu.22635

15. Hernández-Rodríguez AW, Trejo-Medinilla FM. Estudio genético poblacional de frecuencias alélicas para 15 marcadores STR presentes en la población del Estado de Zacatecas aplicado a la práctica forense. Arch Med. 2014;10(1):1. doi:10.3823/1209

16. Bomba L, Walter K, Soranzo N. The impact of rare and low-frequency genetic variants in common disease. Genome Biol. 2017;18(1):77. doi:10.1186/s13059-017-1212-4

17. Zanetti A, D’Avanzo F, Rigon L, et al. Molecular diagnosis of patients affected by mucopolysaccharidosis: A multicenter study. Eur J Pediatr. 2019;178(5):739-753. doi: 10.1007/ s00431-019-03341-8 